rien, Quellen, Völkerrechtssubjekte sowie das Verhältnis von Völkerrecht zu innerstaatlichem Recht informieren ('On the Identification of International Law', S. 157-232). Sanders versucht also nicht eine Einführung in das Völkerrecht, die sich primär an afrikanischem Material orientieren würde oder af rikanischen Anschauungen und Traditionen besonderes Gewicht gäbe, wie man angesichts des Titels erwarten kann. Die insoweit klaffende Lücke, durch Taslim O. Elias nur zum geringen Teil geschlossen, ${ }^{1}$ besteht also nach wie vor.

Davon abgesehen ist positiv zu bemerken, daß Sanders kundig, knapp und präzise in die völkerrechtliche Diskussion einführt. Das Afrika gewidmete Herzstück des Bandes gliedert sich in einen Abschnitt über präkoloniales Afrika, die Kolonialzeit und 'The Africa of Today‘. Die soziologischen Besonderheiten der frühen afrikanischen Gesellschaften werden deutlich gemacht. Die Penetration Afrikas wird als ökonomisch inspiriert geschildert, ihre rechtliche Absicherung kritisch analysiert. Die Kolonialzeit erscheint völkerrechtshistorisch als Episode, Kontinuitäten zwischen altem und heutigem Afrika sucht der Autor allenthalben zu betonen.

Natürlich drängt sich die Frage auf, in welcher Weise der in Südafrika lehrende Sanders zu den die Völkerrechtsdiskussion im Schwarzen Afrika gegenwärtig am meisten bewegenden Fragen der Legitimität von Gewalt und Unterstützung von Gewalt gegen Apartheid, der Menschenrechte, der Rechtslage von Namibia Stellung nimmt: Er klammert diese Fragen weitgehend aus, konstatiert sie gelegentlich (S. 82, 134), weicht aber in die Rolle desjenigen aus, der Probleme nur benennt, Auffassungen anderer mitteilt (gelegentlich undifferenziert: "the African States"), aber selbst nicht Stellung bezieht. Man bemerkt gleichwohl ein bisweilen erstaunliches Einfühlungsvermögen für das Selbstverständnis afrikanischer Staaten und ihre Bemühung um Veränderung völkerrechtlicher Normeninhalte. Fazit danach: ein Buch, das seinem Titel nicht voll gerecht wird, aber kenntnisreich geschrieben ist, das Defizite aufweist, die aus seiner Herkunft erklärbar sind, aber gerade im Hinblick darauf auch durch Offenheit und Feinfühligkeit überrascht.

Philip Kunig

\title{
Margaret Strobel
}

\section{Muslim Women in Mombasa 1890-1975}

Yale University Press, New Haven \& London, 1979, 258 S.

Die hier vorliegende Dissertation ist der Versuch die Geschichte moslemischer Frauen Kenias, und hier vor allem der Küstenstadt Mombasa, darzustellen. Die Schwierigkeit der Untersuchung liegt darin, daß die Autorin sich weitgehend auf mündliche Uberliefe-

1 Vgl. meine Besprechung von T. O. Elias, New Horizons in International Law, VRU 13 (1980), S. 185. 
rungen heute lebender Frauen stützen mußte. Die Grunddaten der geschichtlichen Entwicklung (Umschlagplatz für Sklaven, Ausbau zum wichtigsten Hafen der Region, Kolonialherrschaft der Engländer, Beendigung der Sklaverei, Unabhängigkeit Kenias) sind bisher kaum auf ihre Auswirkungen auf die Sozial- und Gesellschaftsstruktur der Stadt hin überprüft worden. Strobel stellt diesen Wandel der Vielvölkerstadt Mombasa vor allem für die Frauen dar.

Einige relevante Wandlungen sollen hier kurz angesprochen werden: Hatten männliche und weibliche Sklaven bis zur Abschaffung der Sklaverei (1907) einen ähnlich inferioren Status, so änderte sich dieser nach der Befreiung nachteilig für die Frauen. In der etwa 20jährigen Ubergangsphase blieben zwar die älteren ehemaligen Sklaven vor allem bei ihren Familien', da diese ihnen die einzige soziale Sicherheit boten, doch die jüngeren suchten ihre gewonnene Unabhängigkeit zu nutzen. Erheblich eingeschränkt wurde die Freiheit durch die von den Engländern eingeführte Kopfsteuer für Männer. Um diese Abgaben leisten zu können, waren die Männer gezwungen, Arbeiten als Hausdiener anzunehmen, womit die ehemals hier tätigen Sklavinnen verdrängt wurden. Der Hafenausbau förderte auch nur das Arbeitsplatzangebot für Männer. Die arbeitssuchenden Frauen fanden vorwiegend im informalen Sektor Einkommensmöglichkeiten (Prostitution, Kleinhandel, Verkauf von zeitweilig verbotenem Palmwein), da auch die ehemals gemeinsame Arbeit von Mann und Frau in der Landwirtschaft sich jetzt weitgehend auf Männer konzentrierte (vor allem außerhalb Mombasas gültig). Der Markt, der in weiten Teilen Ostafrikas hauptsächlich von Frauen betrieben wurde/wird, liegt in Mombasa fest in der Hand von Asiaten und Hadranis.

Die Zeit nach der Sklaverei überstanden ehemalige Sklavinnen zumeist trotzdem besser als freie Frauen, da sie immer auf sich selbst angewiesen waren. Sie waren von jeher gezwungen, wirtschaftlich aktiv zu sein, während die Frauen von armen freien Familien jetzt oft Arbeiten verrichteten, die sie zuvor zu überwachen hatten. D. h. aber auch, daß der Arbeitsmarkt für die arbeitssuchenden ehemaligen Sklavinnen noch enger geworden war.

Die Frauen wohlhabender freier Familien zogen sich aus dem öffentlichen Leben zurück. Und da sie sowohl durch die Religion als auch durch die Arbeit von den Männern getrennt waren, suchten sie sich ihre Betätigung und Bestätigung im kultischen Bereich, im Veranstaltungsbereich. Elemente ehemalig funktionsfähiger Assoziationen wurden für neue Gemeinsamkeiten in Gruppen herangezogen. Doch bildeten diese Gruppen im Laufe ihrer Entwicklung, die nicht durch Klassen- oder ethnische Restriktionen belastet waren, kein gemeinsames Bewußtsein. Vielmehr befehdeten sie sich offen, um ihre Führerschaft zu dokumentieren. Ein einziges Mal lehnten sich die Gruppen gemeinsam gegen den "Wucher" der Inder auf. Diese ehemaligen Initiations-/Tanzgruppen der Sklavinnen, die diesen einen Zusammenhalt gaben, sind zu Unterhaltungs- und Prestigegruppen herabgesunken. Auch neue Gruppen, die sich integrierende politische Ziele gesetzt haben, sind bis heute nicht in der Lage, ein gemeinsames Bewußtsein unter den Frauen zu entwickeln, da ihre Mitglieder zumeist der gebildeten, wohlhabenderen Schicht angehören und diese Gruppen mehr Wohltätigkeitscharakter haben. 
Die politische und wirtschaftliche Veränderung Mombasas hat den Frauen wenig Möglichkeiten der Teilnahme am Wirtschaftsgeschehen gegeben; z. T. erfolgte eine Verdrängung aus Aufgabenbereichen, z. T. erfolgte aber auch ein Verlust der eigenen Identität, da westlichen Werten der Vorzug gegeben wurde.

Die vorliegende Deskription von Strobel ist deshalb lesenswert, weil hier ein geschichtlicher Ablauf, der den sozialen Wandel vor allem für die Frauen beschreibt, dargestellt wurde. Weniger anerkennenswert ist jedoch, daß für die Beurteilung der Entwicklung der moslemischen Frauen Mombasas das westliche Emanzipationsverständnis zugrunde gelegt wurde.

Gudrun Martius- von Harder

\section{Tony Barnett}

The Gezira Scheme. An Illusion of Development

Frank Cass, London, 1977, 192 S.

Tony Barnett, ein bekannter Sudan-Experte und Mitglied der Overseas Development Group der University of East Anglia, hat hier eine sehr lebendig geschriebene, detaillierte Dorstudie über die sozio-ökonomischen Beziehungen innerhalb eines durchschnittlichen Dorfes mit ca. 3000 Seelen in der Gezira, dem großen Baumwollanbaugebiet zwischen Weißem und Blauem Nil im Sudan, vorgelegt. Sie beruht auf eigenen Beobachtungen und Befragungen, die der Autor zwischen 1970 und 1972 im Sudan machen konnte. Nach Barnett ist das von den Briten zu Beginn des Jahrhunderts angelegte und von den Sudanesen nach der Unabhängigkeit 1955 bruchlos weitergeführte Gezira-Bewässerungs-Projekt, in dem einige 100000 Bauern- und Pächterfamilien auf rund 800000 ha Baumwolle anbauen, nicht als ein zukunftsträchtiges Entwicklungsmodell anzusehen. Obwohl letztlich unklar bleibt, warum der Baumwollanbau keine hinreichenden Multiplikatoreffekte und Vor- und Rückwärtsimpulse auf andere Wirtschaftssektoren ausüben kann, ist diese Studie doch zu den wenigen Feldforschungs-Ergebnissen zu zählen, die unverzichtbar sind, wenn man die gegenwärtigen Entwicklungsprobleme des Sudan begreifen will.

Rainer Tetzlaff 